\title{
MICROARCSECOND VLBI: WHAT WILL WE SEE?
}

\author{
ESKO VALTAOJA \\ Metsähovi Radio Research Station, SF-02540 Kylmälä, Finland
}

\section{INTRODUCTION}

With the exception of some pioneering mm-VLBI observations, the present-day VLBI cannot resolve the most compact components in active galaxies, nor measure their brightness temperatures. In addition, the observing frequencies are too low: the innermost components are still self-absorbed and often completely undetectable at standard VLBI frequencies. However, studies of continuum spectra and variations of AGN can give us estimates of what will eventually be seen when VLBI techniques reach sufficient resolutions and frequencies. These estimates are also useful in considering what capabilities will be required from future interferometry for studying AGN.

We have used multifrequency continuum monitoring data together with standard shock-in-jet models to derive flux, size and brightness temperature distributions for the innermost core components in a complete $2 \mathrm{Jy}$ sample of AGN. The millimeter spectra and its variations can be satisfactorily explained by just two types of high-frequency components, a relativistic inhomogeneous jet and evolving shocks in this jet (Valtaoja et al. 1988). Moreover, with extended multifrequency flux monitoring the contributions from these two components can be separated, which makes possible the extraction of limited amounts of structural data (average sizes, brightness distributions, etc.) just from continuum data.

We have used two main methods. Cm-to-mm spectra can reveal the existence of persistent high frequency components and give estimates of their fluxes and turnover frequencies. Continuum monitoring gives fluxes and variability timescales of transient components, and further their sizes and brightness temperatures.

\section{RESULTS}

We have obtained multiepoch $\mathrm{cm}$-to-mm spectra of a complete sample of bright radio sources using the SEST, Metsähovi, Hartebeesthoek and Itapetinga radio telescopes (Tornikoski et al., preprint). The quiescent millimeter spectra are smooth, steep continuations of cm-spectra: the optically thin radiation from the core dominates, and there is no evidence for the existence of new strong components (at $0.5 \mathrm{Jy}$ level) at 3 or $1.3 \mathrm{~mm}$. The spectra are steep, with average $8.4-90 \mathrm{GHz}$ index $\approx-0.4$ and $90-230 \mathrm{GHz}$ index $\approx-0.7$. Typically, the total flux of any of the $\approx 100$ brightest sources in the sky at $230 \mathrm{GHz}$ will be $<0.5 \mathrm{Jy}$. The brightest and most compact components seen at high frequencies are invariably new shocks, not cores. At best, in some blazars these shocks can transiently raise the mm-fluxes (both total and VLBI) to $\mathrm{cm}$-flux levels. 
Infrared-to-radio monitoring of 3C 273 (Robson et al., preprint) shows that the high-frequency variations are caused by the same evolving shocks as the cmto-mm radio variations; no new mechanisms are apparent, although some shocks appear to die out almost completely before reaching lower radio frequencies.

From continuum monitoring in Metsähovi at 22 and $37 \mathrm{GHz}$ (Teräsranta et al. 1992) we have derived shock component fluxes, timescales and brightness temperatures. The shock component fluxes can be estimated directly from the flux monitoring data as the difference between quiescent flux levels and outburst flux levels. From shock fluxes and observed variability timescales the shock brightness temperatures can be calculated. We find that the characteristic sizes of the shocks $\left(=2 \tau_{v a r}\right)$ in different sources are very similar, $\approx 1$ l.y. $(\approx 80 \mu \mathrm{as}$ at $z=0.5$ ) at the time of $22 \mathrm{GHz}$ maximum flux. The highest brightness temperatures in a few highly polarized quasars and BL Lacs may exceed $10^{13} \mathrm{~K}\left(H_{0}=100 \mathrm{kms}^{-1} / \mathrm{Mpc}\right)$, but are typically $\approx 10^{12} \mathrm{~K}$ in ordinary quasars and $<10^{12} \mathrm{~K}$ in radio galaxies (Teräsranta and Valtaoja, preprint). It is worth emphasizing that the flux, compactness and brightness temperature of a source all are highly time-dependent, and previous VLBI observations cannot be used to predict, for example, the correlated flux (Valtaoja et al. 1992).

Based on our continuum results, a typical bright source at the time of its $22 / 37 \mathrm{GHz}$ outburst maximum consists of two components, a core with $S<1$ Jy, $T_{b}<10^{12} \mathrm{~K}$, and a more compact new shock component with $S>1 \mathrm{Jy}, T_{b}>$ $10^{12} \mathrm{~K}$ and a physical size $\approx 1$ l.y. Assuming a typical superluminal expansion speed $v / c \approx 4$, the component separation is of the order of $100-200 \mu$ as at this time $(z \approx 0.5$, shock age $\approx 0.5$ years $)$.

\section{CONCLUSIONS}

In summary, the predicted largest brightness temperatures and smallest component sizes in the majority of AGN are not very far beyond today's VLBI capabilities. The dominant components in the radio-IR regime are the core-jet and shocks in this jet, with no evidence of other major components in (radioloud) AGN. Thus, the main task of future VLBI in AGN research will probably be to resolve the structure of the shocked synchrotron-radiating jets. In order to do this, one needs to reach $T_{b} \approx 10^{12}-10^{13} \mathrm{~K}$ and $\theta \approx 10 \mu \mathrm{as}$, but not much beyond that. Millimeter and submillimeter VLBI should also have a sensitivity approaching that of today's cm-VLBI due to the general faintness of the AGN components at high frequencies, and the schedulling must be very flexible to catch the rapidly evolving early phases of the shocks.

\section{REFERENCES}

Teräsranta, H., et al. 1992, A\&AS, 94, 121

Valtaoja, E., et al. 1988, $A \mathscr{E} A, \mathbf{2 0 3}, 1$

Valtaoja, E., et al. 1992, $A \mathscr{E} A S, \mathbf{9 5}, 73$ 